\title{
ROLE OF THE REPLICASE GENE OF MURINE CORONAVIRUS JHM STRAIN IN HEPATITIS
}

\author{
Sonia Navas-Martín, Maarten Brom, and Susan R. Weiss*
}

\section{INTRODUCTION}

Mouse hepatitis virus (MHV) is the prototype of group II coronaviruses. Various strains of MHV induce different patterns of pathogenesis. MHV-JHM is a highly neurovirulent strain that causes severe acute encephalitis and chronic demyelination, but not hepatitis. MHV-A59 strain is dualtropic, causing mild to moderate hepatitis, as well as acute meningoencephalitis and chronic demyelination in C57BL/6 mice. Using a combination of targeted RNA recombination to precisely manipulate the coronavirus genome, and in vivo approaches (the mouse model), we have previously reported that the coronavirus spike protein is a major determinant of pathogenesis. ${ }^{1,2}$ Interestingly, we have also found that expression of the "hepatotropic" A59 spike glycoprotein within the background of the "neurotropic" JHM strain does not reproduce the A59 hepatotropic phenotype. ${ }^{3}$ Thus, our studies demonstrated that genes other than the spike play a role in coronavirus tropism and virulence. These results prompted us to further investigate which genes may account for the lack of hepatotropism of the JHM strain. We have started to assess the role of the JHM replicase gene in pathogenesis.

Here, we have generated a recombinant chimeric JHM-A59 virus, in which the whole JHM replicase gene was introduced into the A59 background (JHMrep-RA59). We have performed in vitro replication kinetics analysis, and in vivo studies in order to compare JHMrep-RA59 with RJHM and RA59 (recombinant wild types). In vitro studies demonstrate that the JHMrep-RA59 virus replicates with similar kinetics to RA59. In vivo studies demonstrate that the presence of the 3' third of the A59 genome is sufficient to confer on a recombinant virus the ability to induce hepatitis; thus the replicase gene of JHM strain does not account for the non-hepatotropic phenotype of JHM. Our results suggest that genes other than spike and replicase must play a role in the tropism of JHM.

\footnotetext{
* Sonia Navas-Martín, Drexel University College of Medicine, Philadelphia, Pennsylvania 19102. Maarten Brom, Susan R. Weiss, University of Pennsylvania School of Medicine, Philadelphia, Pennsylvania 19104.
} 


\section{METHODS}

\subsection{Cells, Plasmids, and Viruses}

Murine fibroblast (L2 and 17Cl.1) cells and Felis catus whole-fetus (FCWF) cells were maintained in Dulbecco's minimal essential medium (DMEM) and supplemented with 10\% fetal bovine serum (FBS), 1\% antibiotic-antimycotic (penicillin-streptomycinamphotericin B), and $10 \mathrm{mM}$ HEPES buffer solution. The helper virus AMHV-B3b as well as the plasmid pJHM were provided by Stanley Perlman (University of Iowa). pMH54 plasmid and helper virus fMHV were provided by Paul Masters (Wadsworth Center for Laboratories and Research, New York State Department of Health, Albany, $\mathrm{NY}$ ).

\subsection{Targeted RNA Recombination}

All isogenic recombinant viruses were generated as previously described, ${ }^{4,5}$ using fMHV and pMH54 (for A59 background viruses) and AMHV-JHM 3Bb and pJHM (for JHM background viruses). Isogenic chimeric A59 viruses expressing the replicase gene of the JHM strain were constructed using fMHV-JHM 3Bb as parental virus and pMH54 as donor plasmid (Figure 1). JHM and A59 wild-type recombinant viruses were generated using $\mathrm{fMHV}-\mathrm{JHM} 3 \mathrm{Bb} / \mathrm{pJHM}$, and $\mathrm{fMHV} / \mathrm{pMH} 54$, respectively. Recombinant viruses were selected by their ability to infect murine cells.

\subsection{Viral Load and Histopathology in Liver}

To study virulence, C57BL/6 mice were infected intracranially (IC) with 10-fold serial dilutions of virus, five mice per dilution. Fifty percent lethal dose (LD50) values were calculated as previously described. ${ }^{6}$ In order to assess viral load in the liver, mice were inoculated intrahepatically $(\mathrm{IH})$ with 500 plaque forming units (pfu) of virus as described previously. ${ }^{2}$ Livers were harvested from infected and mock mice on day 5 postinfection (p.i.). A piece of the liver was fixed overnight with $10 \%$ buffered formalin, and the rest of the liver was used for virus titration. Formalin-fixed liver was embedded

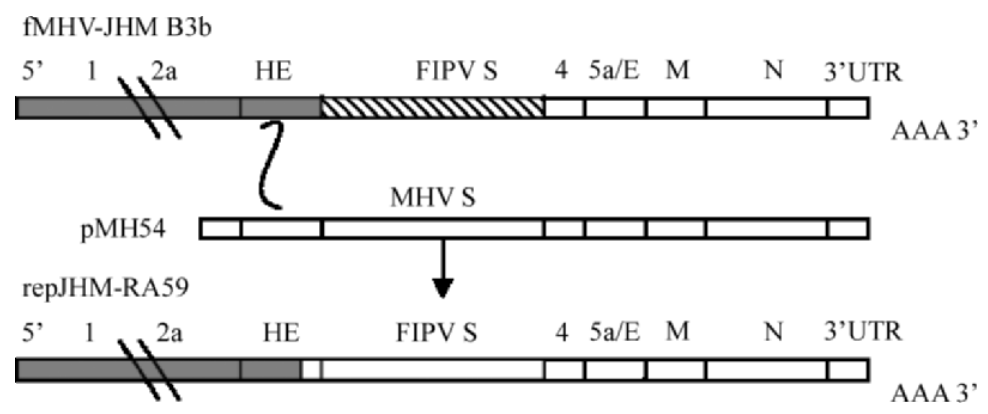

Figure 1. Schematic of targeted RNA recombination. 
in paraffin, sectioned and stained with hematoxylin and eosin (H\&E), and used for pathologic evaluation. Viral load (pfu/g liver) was determined by plaque assay. ${ }^{2}$

\section{RESULTS AND DISCUSSION}

MHV-JHM is a highly neurovirulent strain that does not cause hepatitis in infected mice. This is in contrast with other MHV strains, such as A59, that induce mild to moderate hepatitis. ${ }^{7,8}$ Using isogenic recombinant viruses in the A59 background that differ only in the spike gene, we have previously demonstrated that the spike glycoprotein of murine coronavirus is a major determinant of hepatitis ${ }^{2}$ and CNS disease. ${ }^{1,9}$ However, we have also shown that recombinant viruses in the JHM background that express the spike of the hepatotropic A59 strain (SA59-RJHM) replicate poorly in the liver and do not induce hepatitis, while they do cause encephalitis to an intermediate level, between that of A59 and JHM (Ref. 3 and Iacono et al., mss in preparation). These results were surprising considering the role of the spike glycoprotein in pathogenesis and demonstrate that the JHM genetic background eliminates the ability of the A59 spike to mediate hepatotropism. Therefore, a major question arises from these studies, that is what JHM genes determine its non-hepatotropic phenotype?

Here, we wanted to assess whether the replicase gene of the neurotropic murine coronavirus JHM strain (MHV-JHM), determines its lack of hepatotropism. Using targeted RNA recombination, we have generated chimeric recombinant A59 viruses that express the replicase gene of the non-hepatotropic JHM strain (repJHM-RA59) (Figure 1). We have generated 2 independent chimeric repJHM-RA59 viruses, together with recombinant RA59 and RJHM wild-type viruses. Independent recombinants from each genotype exhibited the same phenotypes in vitro as well as in vivo. The data shown here represent results obtained from one recombinant virus per construct (RA59, RJHM and repJHM-RA59).

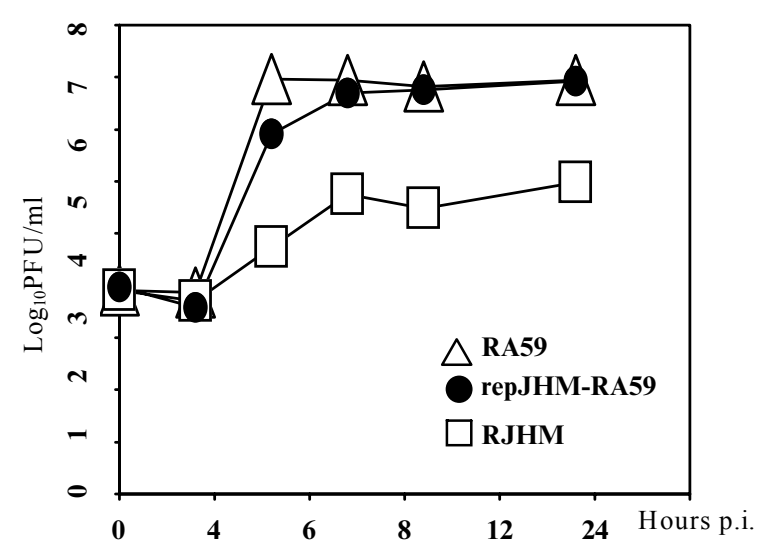

Figure 2. Growth kinetics of MHV recombinant viruses in $\mathrm{L} 2$ cells. Cells were infected at $37^{\circ} \mathrm{C}$ at an MOI of 1 . Titers of released virus in the cultures were measured by plaque assay. 
It is well-known that A59 and JHM viruses exhibit very different replication kinetics in vitro. MHV-A59 replicates to a higher titer, whereas JHM replicates with slower kinetics and to a lower final titer, and displays higher levels of fusion and cytotoxicity. ${ }^{10}$ We have previously reported that RA59 and RJHM recombinant viruses generated by targeted RNA recombination mimic both in vitro as well as in vivo phenotypes of wild type A59 and JHM viruses. ${ }^{3}$ Here, we found that recombinant A59 viruses expressing the replicase gene of JHM, exhibit the same replication kinetics pattern as RA59, whereas RJHM replicates with slower kinetics and to a lower final titer than RA59 and repJHMRA59 (Figure 2). This suggests that, at least in the cells tested, structural genes rather than the replicase have a major role in JHM replication kinetics.

We next evaluated the virulence of repJHM-RA59 compared to wild type recombinants RA59 and RJHM. Interestingly, recombinant A59 viruses expressing the replicase gene of JHM exhibited the same range of virulence values as RA59 $\left(\log _{10} \mathrm{LD}_{50}\right.$ 3.6-3.8), in contrast with the highly neurotropic RJHM (Table 1). Although our study did not address the question of whether virulence factors are encoded by the replicase gene of JHM virus, it demonstrates that chimeric A59 virus expressing the replicase gene of JHM is as virulent as A59 wild-type in mice, and that the virulence is mostly determined by the 3 ' end of the viral genome, suggesting that structural genes have a major role in the highly neurovirulent JHM phenotype.

Finally, we evaluated viral load and hepatitis at day 5 p.i., that is the peak of replication in the liver (Figure 3, Table 2). We did not observe differences in viral load and histopathology in the livers of mice infected with repJHM-RA59 compared with RA59 viruses. repJHM-RA59 as well as RA59 infected mice exhibited mild to moderate hepatitis, in a range that we have previously observed for A59 strain. ${ }^{2,3}$ Viral load correlated with hepatitis in infected mice (data not shown). In contrast, RJHM induces none to minimal changes in the liver and replicates poorly, as expected.

In summary, we have generated recombinant A59 viruses expressing the replicase gene of the neurotropic JHM strain (repJHM-RA59) and compared their in vitro replication kinetics as well as their ability to replicate and induce hepatitis in mice. Our data demonstrate that (1) repJHM-RA59 viruses replicate in vitro to the same extent (load and kinetics) as RA59; (2) repJHM-RA59 viruses are as virulent in mice as A59 wild type; (3) repJHM-RA59 viruses induce hepatitis similarly to RA59.

Our data suggest that the lack of hepatotropism of the JHM strain is not determined by the replicase gene; rather the 3' end of the genome may have a major role in JHM phenotype in the liver. The role of the replicase gene of coronaviruses in pathogenesis

Table 1. Virulence of recombinant viruses after intracranial inoculation.

\begin{tabular}{cc}
\hline Virus & $\begin{array}{c}\log _{10}\left(\mathrm{LD}_{50}\right) \\
\text { i.c. }{ }^{a}\end{array}$ \\
\hline RA59 & $3.6-3.8$ \\
RJHM & 0.8 \\
repJHM-RA59 & $3.6-3.8$ \\
\hline
\end{tabular}

${ }^{\mathrm{a}}$ Intracranial virulence expressed as $\log _{10}\left(\mathrm{LD}_{50}\right)$. 


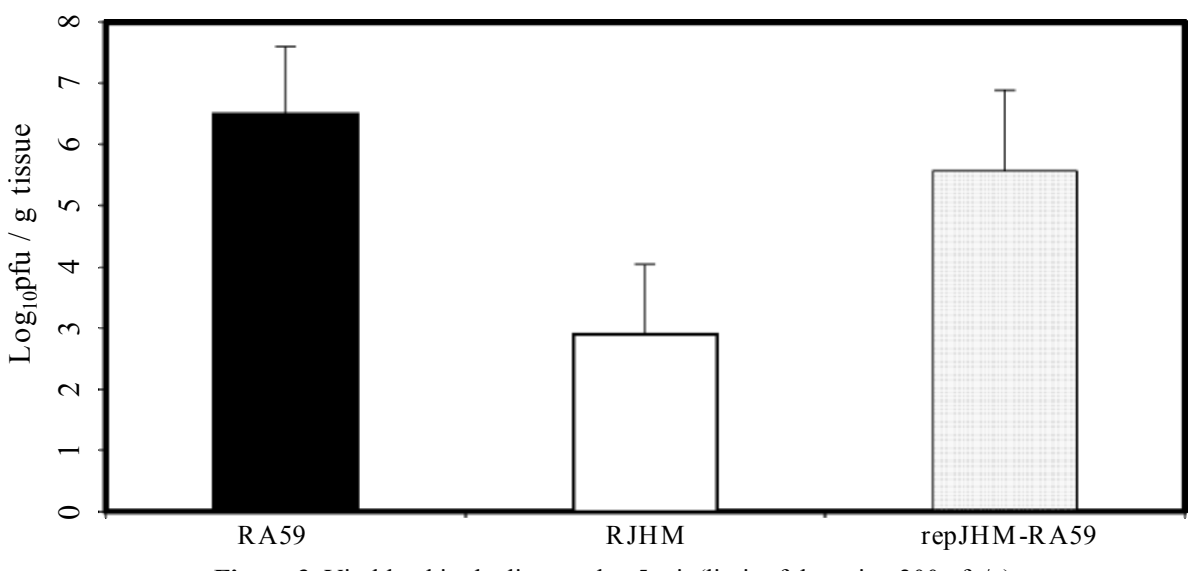

Figure 3. Viral load in the liver at day 5 p.i. (limit of detection $200 \mathrm{pfu} / \mathrm{g}$ ).

remains poorly understood. The replicase gene is approximately $20 \mathrm{~kb}$, and encodes a protein complex of up to 16 viral subunits that together with a number of cellular proteins form the replicase complex. ${ }^{11}$ It should be pointed out that the data generated using these chimeras do not rule out the possibility that replicase proteins influence pathogenesis. In particular, Sperry et al. ${ }^{12}$ have identified mutations in ORF $1 \mathrm{~b}$ (p59-Our data suggest that the lack of hepatotropism of the JHM strain is not determined ns14) and 2a that attenuate virus replication and virulence in mice but do not affect in vitro replication. Their results suggest that proteins of the replicase complex (as well as nonstructural proteins such as 2a) serve roles in pathogenesis distinct from functions in virus replication.

Overall, our data demonstrates that structural genes of JHM may play a major role in coronavirus replication kinetics in vitro, and that the replicase gene of JHM may not determine the lack of hepatotropism of JHM strain.

\section{ACKNOWLEDGMENTS}

This work was supported by NIH grants AI-17418 and AI60021 (formerly NS21954) to S.R.W. S.N.M. is supported by internal funds of Drexel University College of Medicine.

Table 2. Viral induced histopathology in the liver.

\begin{tabular}{lccccc}
\hline \multicolumn{1}{c}{ Virus } & None & Minimal & Mild & Moderate & Severe \\
\hline RA59 & & & $20 \%$ & $60 \%$ & $20 \%$ \\
RJHM & $40 \%$ & $60 \%$ & & & \\
repJHM-RA59 & & & $40 \%$ & $60 \%$ & \\
\hline
\end{tabular}

Results are shown as percentage of mice exhibiting minimal, mild, moderate or severe hepatitis. 


\section{REFERENCES}

1. J. J. Phillips, M. M. Chua, E. Lavi, and S. R. Weiss, Pathogenesis of chimeric MHV4/MHV-A59 recombinant viruses: the murine coronavirus spike protein is a major determinant of neurovirulence, $J$. Virol. 73, 7752-7760 (1999).

2. S. Navas, S. H. Seo, M. M. Chua, J. D. Sarma, E. Lavi, S. T. Hingley, and S. R. Weiss, Murine coronavirus spike protein determines the ability of the virus to replicate in the liver and cause hepatitis, J. Virol. 75 , 2452-2457 (2001).

3. S. Navas and S. R. Weiss, Murine coronavirus-induced hepatitis: JHM genetic background eliminates A59 spike-determined hepatotropism, J. Virol. 77, 4972-4978 (2003).

4. L. Kuo, G. J. Godeke, M. J. Raamsman, P. S. Masters, and P. J. Rottier, Retargeting of coronavirus by substitution of the spike glycoprotein ectodomain: crossing the host cell species barrier, J. Virol. 74, 13931406 (2000).

5. E. Ontiveros, L. Kuo, P. S. Masters, and S. Perlman, Inactivation of expression of gene 4 of mouse hepatitis virus strain JHM does not affect virulence in the murine CNS, Virology 289, 230-238 (2001).

6. L. J. Reed and H. Muench, A simple method of estimating fifty per cent points, Am. J. Hygiene 27, 493-497 (1938)

7. J. Haring and S. Perlman, Mouse hepatitis virus, Curr. Opin. Microbiol. 4, 462-446 (2001).

8. A. E. Matthews, S. R. Weiss, and Y. Paterson, Murine hepatitis virus--a model for virus-induced CNS demyelination, J. Neurovirol. 8, 76-85 (2002).

9. J. Das Sarma, L. Fu, J. C. Tsai, S. R. Weiss, and E. Lavi, Demyelination determinants map to the spike glycoprotein gene of coronavirus mouse hepatitis virus, J. Virol. 74, 9206-9213 (2000).

10. T. M. Gallagher and M. J. Buchmeier, Coronavirus spike proteins in viral entry and pathogenesis, Virology 279, 371-374 (2001).

11. J. Ziebuhr, The coronavirus replicase, Curr. Top. Microbiol. Immunol. 287, 57-94 (2005).

12. S. Sperry, L. Kazi, R. Graham, R. Baric, S. Weiss, and M. Denison, Single amino acid substitutions in nonstructural ORF1b-nsp14 and ORF2a 30kDa proteins of the murine coronavirus MHV-A59 are attenuating in mice, J. Virol. 79, 3391-3400 (2005). 